\title{
Delay-Limited Capacity in the Low Power Regime
}

\author{
Zouheir Rezki and Mohamed-Slim Alouini \\ Computer, Electrical, and Mathematical Sciences and Engineering (CEMSE) Division \\ King Abdullah University of Science and Technology (KAUST), Saudi Arabia \\ \{zouheir.rezki,slim.alouini\}@kaust.edu.sa
}

\begin{abstract}
Outage performance of the $M$-block fading with additive white Gaussian noise (BF-AWGN) is investigated in the low-power regime. We consider delay-constrained constant-rate communications with perfect channel state information (CSI) at both the transmitter and the receiver (CSI-TR), under a shortterm power constraint (STPC) and a long-term power constraint (LTPC). Subject to STPC, we show that selection diversity that allocates all the power to the strongest block is asymptotically optimal. Then, we provide a simple characterization of the outage probability in the regime of interest. We quantify the reward due to CSI-TR over the constant-rate constant-power scheme and show that this reward increases with the delay constraint. For instance, for Rayleigh fading, we find that a power gain up to $4.3 \mathrm{~dB}$ is achievable. Subject to LTPC, we show that the above guidelines still holds and that the outage performance improves due to the flexibility of the LTPC over the STPC. More interestingly, we prove that LTPC allows zero-outage communication even at low SNR and characterize the delaylimited capacity at low SNR in a simple form. More precisely, we establish that the delay-limited capacity scales linearly with the power constraint, for a given $M<\infty$. Our framework highlights the benefit of fading at low SNR as the delay-limited capacity may outperform the AWGN capacity. For instance, for Rayleigh fading and with $M=3$, the delay-limited capacity is $16 \%$ higher than the capacity of an AWGN channel.
\end{abstract}

Index Terms-Outage capacity, low-power regime, blockfading channels, channel state information, selection diversity.

\section{INTRODUCTION}

In many situations, communications should happen in the low-power regime. For instance, wideband communications where the power per sub-band can be very low, sensor network communications, free-space optical communications, etc. The low-power framework is also useful to study communication systems where the power per degrees of freedom (time, space or frequency) is low. A vast body of work exists by now focusing on communications at low-power regime either from performance limits and signaling design perspectives, e.g., [1]-[5], or from more practical views related to deployment, implementation and challenges, e.g., [6], [7], to cite only few. Studying performance limits in the low power regime is also attractive because this regime provides generally the highest energy efficiency defined as the number of bits one can communicate reliably using 1 unit of energy. The latter yardstick is also related to the energy per bit defined as the

This work was funded by a Competitive Research Grant (CRG2) from the Office of Competitive Research Funding (OCRF) at KAUST, URF/1/1695.

Part of this work has been presented at the 2014 IEEE International Symposium on Information Theory (ISIT'2014), Hanolulu, HI, USA, June 2014. energy required to send 1 bit reliably which also hits its minimum in the low power regime.

In delay-unconstrained communication, the ergodic capacity is the most popular performance measure. Characterization of performance limits in this case takes the form of deriving the capacity behavior at asymptotically low-power regime depending on the channel state information (CSI) at the receiver (CSI-R) or/and at the transmitter (CSI-T), e.g., [8][13]. On the contrary, for delay-constrained communications, much fewer papers have focused on performance in the lowpower regime [14], [15]. There exists, however, a considerable number of papers dealing with outage performance and highlighting the effect of fading, e.g., [16]-[19]. A thorough overview of both delay-constrained and delay-unconstrained communications may be found in [20]. Unfortunately, most existing work if not all, only report partial treatment of the low SNR regime. In [15], [21] (see also [22, Chap. 5]), it has been shown that the role of fading on outage performance is much more important in the low-power regime than in the high-power regime thereby strongly motivating performance limits derivations of delay-constrained communications in the low-power regime.

\section{A. Contribution of the paper}

In this paper, which is an enhanced version of [23], we consider delay-constrained communications over a block fading (BF) channel with additive white Gaussian noise (AWGN). We assume that communication should happen within a limited number of blocks $M$ that constitute a frame. Despite the availability of perfect CSI at the transmitter and the receiver (CSI-TR), a constant-rate coding scheme is imposed. With perfect CSI-T, one could adapt the rate to the channel mutual information so that the outage is equal to 0 . However, there is an issue with this strategy. In deep fades, the code rate could be very low or even equal to zero, which is not desirable in real-time applications. To circumvent this issue, the notion of capacity versus outage was developed for constant rate realtime applications for a block-fading channel model [19], [24], [25]. In the capacity versus outage problem, a constant target rate aims to be maintained throughout the fading process. An information outage occurs when the target rate is higher than the instantaneous mutual information [25]. However, Power control is exploited to minimize the outage probability. The maximum target rate that can be achieved with an outage probability less than $\epsilon$ is called the outage capacity, and the zero outage capacity is called the delay-limited capacity in [25]. This problem has been solved in [25]. Nevertheless, as 
we argue next (Section II), the solution does not lend itself to nice analytical formulations, thus preventing gaining insight on the impact of system parameters on outage performance. Our focus is on the low-power regime that is formally defined next. We consider first communications under a short-term power constraint (STPC) and then communication under a long-term power constraint (LTPC).

Subject to STPC, we show that the minimum outage takes a simple form. More precisely, we show that for the setting described above, selection diversity is asymptotically optimal. Furthermore, we highlight the value of CSI-TR over CSI$\mathrm{R}$ in terms of outage probability in function of the number of blocks $M$ and for different types of fading. Perhaps the most striking result of this part is the fact that even under a short-term power constraint, the gain in terms of outage probability due to CSI-TR over CSI-R increases unboundedly with $M$, provided that the targeted outage performance is low. Subject to LTPC, we show that the results obtained under STPC still hold and that for an equal power constraint, the LTPC provides a better outage performance than the STPC. Furthermore, we argue that zero-outage communication is possible under LTPC even at low SNR, and we characterize the delay-limited (also called zero-outage) capacity in this regime. Essentially, the capacity is shown to scale linearly with the power constraint. Selection diversity is once again optimal, but our optimal strategy requires channel inversion to achieve zero-outage performance.

We note that our framework, likewise [25], requires CSI-T of the $M$ channel coefficients prior to communication. However, the $M$ blocks may correspond, for instance, to channels in different non-overlapping frequency bands in a multicarrier system with parallel subchannels [25], or may correspond to the number of distinct eigenvalues in a multiple-antenna communication system after a singular value decomposition (SVD) precoding at the transmitter. In that case, having CSIT of the $M$ fading blocks seems to be feasible. Nevertheless, for large $M$ or/and in case of long term power constraint (LTPC), computing the optimal power profile and the related outage performance becomes cumbersome for an arbitrary (not necessarily small) value of the transmit power. This handicap, however, is avoided in the low power regime since neither the asymptotically optimal power profile under STPC nor the one under LTPC requires all channel gains instantaneously, as we show in the sequel.

\section{B. Outline of the paper}

The paper is organized as follows. Section II describes the system model and provide useful related background. In section III, performance limits in the low-power regime under an STPC are derived. The corresponding gain due to CSI-TR over CSI-R only is characterized in section IV. Performance limits under an LTPC are studied in section V. Selected numerical results are presented in section VI. Finally, section VII concludes the paper.

Notations: The logarithms $\log (x)$ is the natural logarithm of $x$. We say that $f(x) \stackrel{a}{\approx} g(x)$ if and only if $\lim _{x \rightarrow a} \frac{f(x)}{g(x)}=1$. When it is clear from the context, we omit $a$ in $\stackrel{a}{\approx}$ for convenience. The symbols $\gtrsim$ and $\lesssim$ are defined analogously. the function $[x]^{+}$is equal to $x$ if $x$ is positive and 0 otherwise. $\mathbb{R}_{+}^{M}$ designates the $M$-dimensional set of non-negative real numbers. For a nonnegative integer $n, n$ ! represents factorial $n$. $\operatorname{Prob}(\mathcal{A})$ is the probability of the event $\mathcal{A}$. The complement of $\mathcal{A}$ is denoted by $\mathcal{A}^{c} . F^{(n)}(x)$ represent the $n$-derivative of the function $F(\cdot)$ at $x$. Bold face letters represent $M$-dimension vectors, i.e., $\boldsymbol{x}=\left(x_{1}, \ldots, x_{M}\right)$.

\section{System Model AND Related Background}

We consider a block-fading with additive white Gaussian noise (BF-AWGN) channel comprised of $M$ blocks. Each block contains $N$ symbols that undergo the same fading state which is constant, but random. A codeword of length $M_{N}=M N$ spans $M$ blocks. The $n$-th, $n=1, \ldots, N$, channel output at coherence block $m, m=1, \ldots, M$, is expressed by:

$$
y(m, n)=h_{m} x(m, n)+z(m, n),
$$

where $x(m, n) \in \mathbb{R}$ is the $n$-th transmitted symbol at time coherence $m ; h_{m} \in \mathbb{R}$ is a zero-mean and unit-variance channel gain during block $m$ and $z(m, n) \in \mathbb{R}$ is the AWGN with zero-mean and unite-variance. The fading process $\left\{h_{m}\right\}$ is assumed to be independent (not necessarily identically distributed) across the $M$ blocks. We assume perfect CSI-TR. For convenience, we let $\gamma_{m}=\left|h_{m}\right|^{2}, f_{m}(\cdot)$ its probability density function (PDF), and $F_{m}(\cdot)$ its cumulative distribution functions (CDF), $m=1, \ldots, M$. We assume that $F_{m}(\cdot)$ is defined and infinitely differentiable at 0 to invoke Taylor's theorem regarding expansion of $F_{m}(\cdot)$ around 0 . We assume that $N$ is sufficiently large $(N \rightarrow \infty)$ and study performance limits of delay-limited communications at a constant rate $R$, for a given finite number of blocks $M$. Furthermore, the transmitter is constrained by either a short term power constraint (STPC) or a long term power constraint (LTPC) defined, respectively, by [25]:

$$
\begin{aligned}
\frac{1}{M} \sum_{m=1}^{M} \mu_{m} & \leq P, \\
\frac{1}{K} \sum_{k=1}^{K} \frac{1}{M} \sum_{m=1}^{M} \mu_{m}^{(k)} & \leq P,
\end{aligned}
$$

where $\mu_{m}^{(k)}=\mathrm{E}\left[\left|x^{(k)}(m, n)\right|\right]^{2}$, and the last expectation is with respect to the distribution of the channel input $x^{(k)}(m, n)$, for all $n=1, \ldots, N$; and $K$ is the length of the $M$-block frames sequence. The maximum mutual information of this channel is given by [25]:

$$
I_{M}(\boldsymbol{\gamma}, \boldsymbol{\mu})=\frac{1}{M} \sum_{m=1}^{M} \frac{1}{2} \log \left(1+\gamma_{m} \mu_{m}\right) .
$$

The outage probability computed at a rate $R$ is given by:

$$
P_{\text {out }}^{C S I-T R}(R, P, M)=\operatorname{Prob}\left\{I_{M}(\gamma, \mu)<R\right\} .
$$

To distinguish performances under STPC from their counterparts under LTPC, we use the acronyms "st" and "lt" to designate short-term and long-term performance measures, respectively. As in the sequel, we discuss performances when 
only CSI-R is available; we also use the acronyms CSI-TR and CSI-R as superscripts on the outage probability to distinguish between the two cases. In case of CSI-TR, $\boldsymbol{\mu}^{s t}$ and $\boldsymbol{\mu}^{l t}$ depend on $\gamma$, i.e., $\boldsymbol{\mu}^{s t}=\boldsymbol{\mu}^{s t}(\gamma)$ and $\boldsymbol{\mu}^{l t}=\boldsymbol{\mu}^{l t}(\gamma)$. Then, it is of interest to find the optimal power policy that minimizes the outage probability given by (5).

The minimum outage probability of the BF-AWGN channel has been solved (even in a broader scope) in [25]. We outline briefly the related solution under STPC. Let the region $Q$ be defined as $Q=\left\{\gamma_{h} \in \mathbb{R}_{+}^{M}: \gamma_{1} \geq \ldots \geq \gamma_{M}\right\}$. Assume that $\gamma \in$ $Q$, then the optimal power over the $m$-th block, $m=1, \ldots, M$, is given by:

$$
\mu_{m}^{s t}(\gamma)=\left[\lambda^{s t}(b, \gamma)-\frac{1}{\gamma_{m}}\right]^{+},
$$

where $\lambda^{s t}(b, \gamma)=\frac{1}{b} \sum_{l=1}^{b} \frac{1}{\gamma_{l}}+\frac{M}{b} P$ and $b$ is the unique integer in $\{1, \ldots, M\}$ such that $1 / \gamma_{m} \leq \lambda^{s t}(b, \gamma)$ for $m<b+1$ and $1 / \gamma_{m}>\lambda^{s t}(b, \gamma)$ for $m \geq b+1$. In the previous formulation, $b$ represents the number of blocks for which a positive instantaneous power is allocated. Indeed, the optimum power profile in (6) has a water-filling interpretation in the sense that a higher channel gain gets more power than a lower one and very poor channels get no power at all. The optimal outage region $\mathcal{U}(R, P)$ is derived similarly by partitioning $Q$ into $M$ sub-regions $\mathcal{V}_{m} \subset Q$ such that if $\gamma \in \mathcal{V}_{m}$, exactly $m$ blocks are given positive power. The intersection of $\mathcal{U}(R, P)$ with $\mathcal{V}_{m}, m=1, \ldots, M$, is given explicitly by:

$$
\mathcal{U}(R, P) \cap \mathcal{V}_{m}=\left\{\gamma \in Q: \frac{1}{M} \sum_{l=1}^{m} \frac{1}{2} \log \left(\gamma_{l} \lambda^{s t}(m, \gamma)\right)<R\right\} .
$$

To extend the optimal power and the outage region given by (6) and (7), respectively, beyond $Q$ to $\mathbb{R}_{+}^{M}$, one may sort the component of $\gamma$ in a non-increasing order, apply the above result to the sorted fading vector and apply the inverse permutation to the obtained power allocation vector and outage probability region.

The minimum outage probability under LTPC can be solved similarly as the one under STPC described above, but using $s^{*}$ instead of $P$, where $s^{*}$ is obtained by satisfying the LTPC with equality. Specifically, one can define first the power policy $\left\{p^{l t}(\gamma)\right\}$ by:

$$
p_{m}^{l t}(\gamma)=\left[\lambda^{l t}(b, \gamma)-\frac{1}{\gamma_{m}}\right]^{+},
$$

for $m=1, \ldots, M$, where $\lambda^{l t}(b, \gamma)=\left(\frac{e^{2 M R}}{\prod_{l=1}^{b} \gamma_{l}}\right)^{1 / b}$ and where $b$ is the unique integer in $\{1, \ldots, M\}$ such that $1 / \gamma_{m} \leq \lambda^{l t}(b, \gamma)$ for $m<b+1$ and $1 / \gamma_{m}>\lambda^{l t}(b, \gamma)$ for $m \geq b+1$. Then, the optimal power under LTPC is given by:

$$
\mu^{l t}(\gamma)= \begin{cases}p^{l t}(\gamma) & \text { if } \gamma \in \mathcal{R}\left(s^{*}\right) \\ 0 & \text { else }\end{cases}
$$

where $s^{*}$ is defined by:

$$
s^{*}=\sup \{s: \mathcal{P}(s)<P\},
$$

with $\mathcal{P}(s)$ defined by: $\mathcal{P}(s) \triangleq \int_{\mathcal{R}(s)}\left(\frac{1}{M} \sum_{m=1}^{M} p_{m}^{l t}(\gamma)\right) d F(\gamma)$, and $\mathcal{R}(s)$ being the region defined by:

$$
\mathcal{R}(s)=\left\{\gamma \in \mathbb{R}_{+}^{M}: \frac{1}{M} \sum_{m=1}^{M} p_{m}^{l t}(\gamma) \leq s\right\} .
$$

Clearly, $\mathcal{R}\left(s^{*}\right)$ in (9) represents the no-outage region. If $s^{*}$ in (10) is equal to $+\infty$, the no-outage region becomes $\mathcal{R}\left(s^{*}\right)=\mathbb{R}_{+}^{M}$ and the outage probability is equal to 0 . This case is of particular interest as it allows characterizing the socalled delay-limited capacity (also called zero-outage capacity) $C_{0}(P, M)$ as the solution of:

$$
C_{0}(P, M)=\underset{R \geq 0}{\arg }\left\{\underset{\gamma}{\mathrm{E}}\left[\frac{1}{M} \sum_{m=1}^{M} p_{m}^{l t}(\gamma)\right]=P\right\} .
$$

Note that $s^{*}$ may be regarded as an equivalent STPC induced by the LTPC. Intuitively, for a given power $P$, the minimum outage probability under LTPC offers more flexibility in spending the power than its counterpart under STPC. Hence, it is expected that $s^{*}$ be larger than $P$ which translates into a lower outage probability under LTPC than under STPC.

\section{Performance Limits in the Low-Power Regime Under A Short-Term Power Constraint}

From the previous section, it is clear that beyond $M=2$, there is no hope to derive the outage probability in a simple form. The latter difficulty, unfortunately, prevents gaining a useful insight into the behavior of the outage performance with respect to system parameters. We found here another motivation for us to look at the outage performance in the low-power regime.

The low-power regime is captured by letting $P \rightarrow 0$. In delay-unconstrained communication, this is enough since the reliability (arbitrary low decoding probability) is intrinsically contained in the definition of the channel capacity. However, for delay-constrained communications, since the capacity (in Shannon sense) is generally equal to zero, ${ }^{1}$ studying the lowpower regime can only be meaningful if the targeted outage performance is low in order to guarantee a certain reliability. A simple manipulation of (5) provides:

$$
\begin{aligned}
P_{\text {out }}^{\text {st. } C S I-T R}(R, P, M) & \geq \operatorname{Prob}\left\{\frac{1}{2} \log \left(1+\frac{1}{M} \sum_{m=1}^{M} \gamma_{m} \mu_{m}\right)<R\right\} \\
& \geq \operatorname{Prob}\left\{\frac{1}{2} \log \left(1+\frac{1}{M} \sum_{m=1}^{M} \gamma_{\max } \mu_{m}\right)<R\right\} \\
& \geq \operatorname{Prob}\left\{\frac{1}{2} \log \left(1+\gamma_{\max } P\right)<R\right\} \\
& \geq \operatorname{Prob}\left\{\frac{1}{2} \gamma_{\max } P<R\right\} \\
& =F_{\max }\left(\frac{2 R}{P}\right),
\end{aligned}
$$

\footnotetext{
${ }^{1}$ This might not be the case for a class of fading if CSI-T is available. However, in that case, the outage would be equal to zero regardless.
} 
where (13) follows because $\frac{1}{M} \sum_{m=1}^{M} \frac{1}{2} \log \left(1+\gamma_{m} \mu_{m}\right) \leq$ $\frac{1}{2} \log \left(1+\frac{1}{M} \sum_{m=1}^{M} \gamma_{m} \mu_{m}\right)$ due to the Jensen's inequality; (14) holds since $\gamma_{\max }=\max _{m=1}^{M} \gamma_{m}$; (15) is a consequence of the STPC; to obtain (16), we used the inequality $\log (1+x) \leq x$ and finally $F_{\max }(\cdot)$ in (17) is the CDF of $\gamma_{\max }$. From (17), it is clear that if $P \rightarrow 0$ and $R$ is arbitrary (not necessarily small), then the outage probability is arbitrary close to 1 . Therefore, in delay-constrained communications, and under STPC, reliability at low-power regime can only be obtained if $R \rightarrow 0$ too. Hence, with STPC, the low-power regime we are interested in corresponds to the situation where $P, R$ and the outage performance tend all toward 0 . We are now ready to state our main result.

Theorem 1: For the block-fading channel described by (1), with perfect CSI-TR, and subject to STPC (2), the outage performance is characterized as follows:

$$
P_{\text {out }}^{\text {st, } C S I-T R}(R, P, M) \stackrel{R \rightarrow 0}{\approx} \prod_{m=1}^{M} F_{m}\left(\frac{2 R}{P}\right) .
$$

Proof:

Since the lower bound (17) holds true for arbitrary values of $R$ and $P$, not necessarily low, then to establish Theorem 1, it suffices to show the existence of a feasible communication scheme that achieves the lower bound (17). It turns out that selection diversity achieves (17) asymptotically. To see this, let us consider a selection diversity that allocates to a block $m, m=1, \ldots, M$, a power $P_{m}^{*, s t}$ such that

$$
P_{m}^{*, s t}(\gamma)= \begin{cases}M P & \text { if } \quad \gamma_{m}=\max _{1 \leq k \leq M} \gamma_{k} \\ 0 & \text { otherwise. }\end{cases}
$$

The corresponding outage probability is equal to

$$
\begin{aligned}
P_{S D}^{s t, C S I-T R}(R, P, M) & =\operatorname{Prob}\left\{\frac{1}{2 M} \log \left(1+M P \gamma_{\max }\right)<R\right\} \\
& =F_{\text {max }}\left(x_{1}\right) \\
& \stackrel{R \rightarrow 0}{\approx} F_{\max }\left(\frac{2 R}{P}\right)
\end{aligned}
$$

where $x_{1}$ in (21) is equal to $x_{1}=\frac{e^{2 M R}-1}{M P}$ and where the asymptotic equality (22) holds true since $R \rightarrow 0$ (please see Appendix A). Hence, the lower bound is asymptotically achievable, and Theorem 1 is thus proved.

Few remarks are worthwhile.

Remark 1: For the class of fading such that $F_{m}(x)>0$ for all $x>0, m=1, \ldots, M$ (this is the case of most popular fading in wireless communications), since $P_{\text {out }}^{s t, C S I-T R}(R, P, M) \rightarrow 0$, then Theorem 1 implies that we necessarily have $\frac{R}{P} \rightarrow 0$. That is, for this class of fading, reliable communication (communication with arbitrary low outage probability) at low-power regime requires not only that the rate $R$ be small $(R \rightarrow 0)$, but more stringently that the ratio $\frac{R}{P}$ be small too $\left(\frac{R}{P} \rightarrow 0\right)$.

Remark 2: While it is well-known that increasing $M$ can only improve outage performance (assuming optimal coding), Theorem 1 describes how the outage probability decreases with $M$. In fact, if the number of blocks over which communication is happening increases from $M$ to $M+1$, the outage probability decreases by a factor of $F_{M+1}\left(\frac{2 R}{P}\right)$. More specifically, one can express the outage probability in a more explicit way as follows: ${ }^{2}$,

$$
\begin{aligned}
\log \left(P_{\text {out }}^{\text {stCSI-TR }}(R, P, M)\right) & \approx \log \left(\prod_{m=1}^{M} F_{m}\left(\frac{2 R}{P}\right)\right) \\
& \approx \log \left(\prod_{m=1}^{M} \frac{F_{m}^{\left(n_{m}\right)}(0)}{n_{m} !}\left(\frac{2 R}{P}\right)^{n_{m}}\right) \\
& \approx \log \left(\frac{2 R}{P}\right) \sum_{m=1}^{M} n_{m}, \\
& =-\log \left(\frac{P}{2 R}\right) \sum_{m=1}^{M} n_{m}
\end{aligned}
$$

where $n_{m}$ is the smallest integer such that $F_{m}^{\left(n_{m}\right)}(0) \neq 0$, $m=1, \ldots, M$; (24) is obtained by series expansion of $F_{m}(x)$ around zero and because $\frac{R}{P} \rightarrow 0$ (see Remark 1) and (25) follows because $\lim _{\frac{R}{P} \rightarrow 0} \frac{\log \left(\frac{F_{m}^{\left(n_{m}\right)}(0)}{n_{m} !}\right)}{n_{m} \log \left(\frac{2 R}{P}\right)}=0$. Since in (24) Taylor expansion is performed to the $n_{m}$ th order, $m=1, \ldots, M$, then the error incurred by such an approximation goes faster to 0 than $\left(\frac{2 R}{P}\right)^{\sum_{m=1}^{M} n_{m}}$ as $\frac{2 R}{P} \rightarrow 0$. For fixed $P$ and $R$, (26) describes how the outage probability depends on $M$. Note that since $n_{m} \geq 1$, for all $m=1, \ldots, M$, then $\sum_{m=1}^{M} n_{m} \geq M$. Also, because $\frac{P}{2 R} \rightarrow \infty$, then $\sum_{m=1}^{M} n_{m}$ may be regarded as the diversity order provided by coding over $M$ blocks at low-power regime.

Remark 3: The result in Theorem 1 translates directly to a characterization of the so-called outage capacity. Indeed, along similar steps as Remark 2, it is easy to verify that the outage capacity $C_{\epsilon}(\cdot)$ is given by:

$$
C_{\epsilon}(P, M) \approx \frac{1}{2}\left(\prod_{m=1}^{M} \frac{n_{m} !}{F_{m}^{\left(n_{m}\right)}(0)}\right)^{\frac{1}{\sum_{m=1}^{M} n_{m}}} \epsilon^{\frac{1}{\sum_{m=0}^{M-1} n_{m}}} P .
$$

The ratio $\frac{C_{\epsilon}(P, M)}{P}$, defined as the capacity per unit power for a given outage probability $\epsilon$, is a key performance measure in the regime of interest [21]. Therefore, our result also characterizes the capacity per unit power through (27).

We conclude this section by mentioning that although, in the low-power regime, channel estimation might be challenging, the optimal scheme given by (19) does not perform power adaptation. Indeed, the optimal scheme only requires few feedback bits in each frame of $M$ blocks to designate the block with the highest channel gain. More precisely, it needs exactly $\left\lceil\log _{2}(M)\right\rceil$ feedback bits, where $\lceil\cdot\rceil$ is the ceiling function. A question of interest, in this case, would be to assess whether the additional gain provided by CSI-TR over CSI-R is worth the above low rate feedback mechanism. This is answered in Section IV.

\footnotetext{
${ }^{2}$ Note that we are computing the outage probability in the log scale in order to show the diversity order. Also note that although our focus is on low-power regime, the diversity order is still meaningful since the outage probability is arbitrary driven to zero as a consequence of the fact that $\frac{R}{P} \rightarrow 0$.
} 


\section{How Rewarding Perfect CSI At The transmitter is?}

We first evaluate the outage probability assuming perfect CSI-R and no CSI-T. Recall that with CSI-R and under an STPC, the outage probability (using constant power over the blocks) is given by [24]:

$$
\begin{aligned}
P_{\text {out }}^{\text {stCCS }-R}(R, P, M) & =\operatorname{Prob}\left\{\frac{1}{M} \sum_{m=1}^{M} \frac{1}{2} \log \left(1+P \gamma_{m}\right)<R\right\} \\
& \approx \operatorname{Prob}\left\{\sum_{m=1}^{M} \gamma_{m}<\frac{2 M R}{P}\right\}, \\
& =F_{\Sigma}\left(\frac{2 M R}{P}\right),
\end{aligned}
$$

where (29) follows because $\log (1+x) \approx x$ around zero, and where $F_{\Sigma}(\cdot)$ is the $\mathrm{CDF}$ of $\sum_{m=1}^{M} \gamma_{m}$. Note that the right hand side (RHS) of (29) is asymptotically equal to the outage probability of a repetition diversity with constantpower transmission. Hence, with CSI-R, repetition diversity with constant-power transmission is an optimal strategy at asymptotically low-power regime.

There is apparently no explicit expression of $F_{\Sigma}(\cdot)$ for general fading distributions of $\gamma_{m}, m=1, \ldots, M$. Nevertheless, we have the following result that characterizes the gain in terms of outage performance due to CSI-TR.

Theorem 2: Let $n_{m}$ be the smallest integer such that $F_{m}^{\left(n_{m}\right)}(0) \neq 0, m=1, \ldots, M$. Then, we have:

$$
\lim _{P \rightarrow 0} \frac{P_{\text {out }}^{\text {st,CSI-TR }}(R, P, M)}{P_{\text {out }}^{s t, C S I-R}(R, P, M)}=\frac{1}{M^{\sum_{m=1}^{M} n_{m}}} \frac{\left(\sum_{m=1}^{M} n_{m}\right) !}{\prod_{m=1}^{M} n_{m} !} .
$$

Proof: The proof is presented in Appendix B.

Since the availability of CSI-T can only improve outage performance $P_{o u t}^{s t, C S I-R}(R, P, M)$, one needs to verify that the right-hand side (RHS) of (31) is at most equal to 1 . This is not very clear at first glance. However, we show below that this is, in fact, a direct consequence of the following lemma that provides a more general result.

Lemma 1: Let $n$ be a positive integer. Let $V_{n}$ be the simplex defined by:

$$
V_{n}=\left\{\left(t_{1}, \ldots, t_{n}\right) \in[0, \infty)^{n}: \sum_{k=1}^{n} t_{k} \leq 1\right\} .
$$

Then, for any $\left(a_{1}, \ldots, a_{n}\right) \in(0, \infty)^{n}$, we have:

$$
\frac{\Gamma\left(1+a_{1}+\cdots+a_{n}\right)}{\Gamma\left(1+a_{1}\right) \cdots \Gamma\left(1+a_{n}\right)} \leq n^{\sum_{k=1}^{n} a_{k}},
$$

where $\Gamma(\cdot)$ is the Gamma function defined by: $\Gamma(x)=$ $\int_{0}^{\infty} e^{-t} t^{x-1} \mathrm{~d} t$, for $x>0$.

Proof: We first recall a multidimensional integral property of the Gamma function [26]:

$$
\int_{V_{n}} t_{1}^{a_{1}-1} \cdots t_{n}^{a_{n}-1} \mathrm{~d} t_{1} \cdots \mathrm{d} t_{n}=\frac{\Gamma\left(a_{1}\right) \cdots \Gamma\left(a_{n}\right)}{\Gamma\left(1+a_{1}+\cdots+a_{n}\right)}
$$

Then, we need to lower bound the left hand side (LHS) of (33). For this purpose, we let $X_{k}$ be a random variable with PDF $f_{X_{k}}(t)=a_{k} t^{a_{k}-1}$, for $t \in[0,1], k=1, \ldots, n .^{3}$ We also

\footnotetext{
${ }^{3}$ Note that if for some $k, a_{k} \in(0,1)$, then the support of $X_{k}$ can be restricted to $(0,1]$ instead of $[0,1]$, without any consequence on the proof.
}

assume that $X_{k}$ 's are jointly independent such that their joint PDF $f_{\left(X_{1}, \ldots, X_{n}\right)}\left(t_{1}, \ldots, t_{n}\right)=\prod_{k=1}^{n} f_{X_{k}}\left(t_{k}\right)$. The LHS of (33) can be lower bounded as follows:

$$
\begin{aligned}
& \int_{V_{n}} t_{1}^{a_{1}-1} \cdots t_{n}^{a_{n}-1} \mathrm{~d} t_{1} \cdots \mathrm{d} t_{n} \\
= & \frac{1}{\prod_{k=1}^{n} a_{k}} \int_{V_{n}} \prod_{k=1}^{n} f_{X_{k}}\left(t_{k}\right) \mathrm{d} t_{1} \cdots \mathrm{d} t_{n} \\
= & \frac{1}{\prod_{k=1}^{n} a_{k}} \int_{V_{n}} f_{\left(X_{1}, \ldots, X_{n}\right)}\left(t_{1}, \ldots, t_{n}\right) \mathrm{d} t_{1} \cdots \mathrm{d} t_{n} \\
= & \frac{1}{\prod_{k=1}^{n} a_{k}} \operatorname{Prob}\left\{\sum_{k=1}^{n} X_{k} \leq 1\right\} \\
\geq & \frac{1}{\prod_{k=1}^{n} a_{k}} \operatorname{Prob}\left\{X_{k} \leq \frac{1}{n}, k=1, \ldots, n\right\} \\
= & \frac{1}{\prod_{k=1}^{n} a_{k} n^{a_{k}}} .
\end{aligned}
$$

Since for any $x>0, \Gamma(1+x)=x \Gamma(x)$, (32) follows from (33) and (38) immediately.

Finally, we note that the RHS of (31) is indeed smaller than one because:

$$
\frac{1}{M^{\sum_{m=1}^{M} n_{m}}} \frac{\left(\sum_{m=1}^{M} n_{m}\right) !}{\prod_{m=1}^{M} n_{m} !}=\frac{1}{M^{\sum_{m=1}^{M} n_{m}}} \frac{\Gamma\left(1+n_{1}+\ldots+n_{M}\right)}{\Gamma\left(1+n_{1}\right) \ldots \Gamma\left(1+n_{M}\right)} \leq 1,
$$

where the last inequality follows from Lemma 1.

There is no particular difficulty in evaluating the RHS of (31) for given fading channels. For example, for Rayleigh fading, $n_{m}=1, m=1, \ldots, M$, and the RHS of (31) is equal to $\frac{M !}{M^{M}}$. The later result holds for any fading channel that satisfies $n_{m}=1, m=0, \ldots, M-1$. Since $\frac{M !}{M^{M}}$ is strictly decreasing in $M,{ }^{4}$ then it follows from Theorem 2 that for this class of fading, increasing $M$ strictly increases the gap between $P_{\text {out }}^{\text {st,CSI-TR }}(R, P, M)$ and $P_{\text {out }}^{s t, C S I-R}(R, P, M)$. More generally, for all fading channels such that $n_{m}$ is a constant, $m=1, \ldots, M$, we have the following.

Corollary 1: Let $n_{m}$ be the smallest integer such that $F_{m}^{\left(n_{m}\right)}(0) \neq 0$. Then, if $\forall m=1, \ldots, M, n_{m}=n$, then we have:

i.

$$
\lim _{P \rightarrow 0} \frac{P_{\text {out }}^{s t, C S I-T R}(R, P, M)}{P_{\text {out }}^{s t, C S I-R}(R, P, M)}=\frac{(M n) !}{M^{n M}(n !)^{M}},
$$

ii. the ratio $\frac{(M n) !}{M^{n M}(n !)^{M}}$ is strictly decreasing in $M$, for any $n \geq 1$ and thus increasing $M$ strictly enhances the advantage of CSI-TR over CSI-R, in term of outage probability,

iii. the ratio $\frac{(M n) !}{M^{n M}(n !)^{M}}$ converges to 0 as $M \rightarrow \infty$ and hence the advantage of CSI-TR over CSI-R is unbounded.

Proof: Property i in Corollary 1 is an immediate consequence of Theorem 2 and the fact that $n_{m}=n$, for all $m=0, \ldots, M-1$. To prove ii, we recall first the Stirling's formula [27]:

$$
1 \leq \frac{k !}{\sqrt{2 \pi k}\left(\frac{k}{e}\right)^{k}} \leq \frac{e}{\sqrt{2 \pi}},
$$

\footnotetext{
${ }^{4}$ This is because $\frac{(M+1) !}{(M+1)^{M+1}} \frac{M^{M}}{M !}=\frac{M+1}{(M+1)^{M+1}} M^{M}=\left(\frac{M}{M+1}\right)^{M} \leq 1$.
} 
for any integer $k \geq 1$. Then, we let $U(M)=\frac{(M n) !}{M^{n M}(n !)^{M}}$ and use (41) to upper bound $U(M)$ as follows:

$$
1 \leq \frac{U(M)}{\left(\frac{n}{e}\right)^{M n} \frac{\sqrt{2 \pi M n}}{(n !)^{M}}} \leq \frac{e}{\sqrt{2 \pi}} .
$$

Now, with the help of (42), one can upper bound $\frac{U(M+1)}{U(M)}$ as follows:

$$
\begin{aligned}
\frac{U(M+1)}{U(M)} & \leq \frac{e}{\sqrt{2 \pi}}\left(\frac{n}{e}\right)^{(M+1) n} \frac{\sqrt{2 \pi(M+1) n}}{(n !)^{(M+1)}} \frac{1}{\left(\frac{n}{e}\right)^{M n} \frac{\sqrt{2 \pi M n}}{(n !)^{M}}} \\
& =\frac{e}{\sqrt{2 \pi}}\left(\frac{n}{e}\right)^{n} \sqrt{\frac{M+1}{M}} \frac{1}{n !} \\
& \leq \frac{e}{\sqrt{2 \pi}} \sqrt{\frac{M+1}{M}} \frac{1}{\sqrt{2 \pi n}} \\
& \leq \frac{e}{2 \pi} \sqrt{\frac{M+1}{M}} \\
& \leq \frac{e}{2 \pi} \sqrt{2} \\
& <1
\end{aligned}
$$

where (45) is obtained using the LHS of (41); (46) holds because $n \geq 1$ and (47) follows from the fact that the function $x \mapsto \sqrt{\frac{x+1}{x}}$ is monotonically decreasing and thus for all $M \geq 1$, $\sqrt{\frac{M+1}{M}} \leq \sqrt{\frac{1+1}{1}}=\sqrt{2}$. Therefore, $U(M)$ is strictly increasing in $M$ and property ii is thus proved. Finally, in order to verify iii, we again use (42) and observe that:

$$
\begin{aligned}
U(M) & \leq \frac{e}{\sqrt{2 \pi}}\left(\frac{n}{e}\right)^{M n} \frac{\sqrt{2 \pi M n}}{(n !)^{M}} \\
& =e\left(\frac{\left(\frac{n}{e}\right)^{n}}{n !}\right)^{M} \sqrt{n} \sqrt{M} \\
& \leq e\left(\frac{1}{\sqrt{2 \pi n}}\right)^{M} \sqrt{n} \sqrt{M},
\end{aligned}
$$

where (51) follows from the LHS of (41). Clearly, the RHS of (51) converges to zero as $M \rightarrow \infty$ and since $U(M)$ is nonnegative, then $\lim _{M \rightarrow \infty} U(M)=0$. Hence, property iii holds true and Corollary 1 is proved.

Note that Corollary 1 encompasses the important particular case where the fading over the $M$ blocks are identically distributed since, in that case, $n_{m}$ would be constant for all $m=1, \ldots, M$. Furthermore, in light of Corollary 1, it can be inferred that the advantage of CSI-TR over CSI-R can be tremendous for $M$ sufficiently large, at low-power regime, provided that the targeted outage performance (or equivalently the rate $R$ at which we communicate) is low. For instance, using (18), the power required to achieve an outage performance $\epsilon$ while communicating at a rate $R$ assuming CSITR is asymptotically equal to:

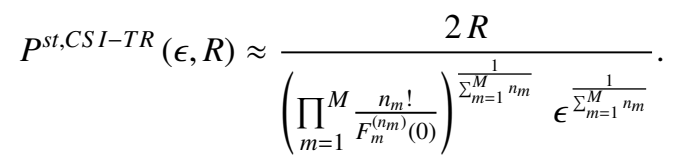

Whereas with CSI-R only, the required power is equal to: ${ }^{5}$

$$
P^{s t, C S I-R}(\epsilon, R) \approx \frac{2 M R}{\left(\frac{\left(\sum_{m=1}^{M} n_{m}\right) !}{\prod_{m=1}^{M} F_{m}^{\left(n_{m}\right)}(0)}\right)^{\frac{1}{\sum_{m=1}^{M} n_{m}}} \epsilon^{\frac{1}{\sum_{m=1}^{M} n_{m}}} .}
$$

Hence, the power gain due to CSI-TR is equal to:

$$
\frac{P^{s t, C S I-T R}(\epsilon, R)}{P^{s t, C S I-R}(\epsilon, R)} \approx\left(\frac{\left(\sum_{m=1}^{M} n_{m}\right) !}{M^{\sum_{m=1}^{M} n_{m}} \prod_{m=1}^{M} n_{m} !}\right)^{\frac{1}{\sum_{m=1}^{M} n_{m}}} .
$$

Now, if $M=2$ and $n_{m}=1$, for all $m=1, \ldots, M$, (54) yields $1.5 \mathrm{~dB}$ gain which is considerable in the low-power regime. Increasing $M$ to 3 enhances further the gain to about 2.18 $\mathrm{dB}$. Actually, it can be easily shown that as $M \rightarrow \infty$, the ratio $\frac{P^{s, C S I-T R}(\epsilon, R)}{P^{s t, C S I-R}(\epsilon, R)}$ converges to $e^{-1}$ and hence for $M$ sufficiently large and assuming all $n_{m}=1$, a remarkable $4.3 \mathrm{~dB}$ gain is achievable. Note that there is no contradiction between the fact that the advantage of CSI-TR over CSI-R in terms of outage probability is unbounded as stated by iii in Corollary 1 and the fact that the power gain resulting from CSI-T is bounded. This is because the exponent in (54) also depends on $M$. Therefore, the common belief by which "optimal power allocation under the short-term constraint does not provide any significant gain with respect to constant power" is somehow misleading. In [25], the authors pointed out that for Rayleigh fading channels, "As $M$ increases, the outage probability with the short-term constraint is always very close to that for constant power". This guideline is unfortunately not very precise and hence should be looked at more carefully, at least in the low-power regime. Indeed, as stated by Corollary 1, the outage probability with the short-term constraint can be arbitrary smaller than that of constant power, for large $M$.

So far, we have focused on the case where all $n_{m}$ are equal, i.e., $n_{m}=n$ is constant and fixed, and we have analyzed the value of CSI-TR over CSI-R in function of $M$. Another aspect of interest would be to fix $M$, and study the reward provided by CSI-TR versus $n$. This would help understanding the value of CSI-TR for different fading channels, for a given delay constraint. For convenience, our result is summarized in the following corollary.

Corollary 2: Let $n_{m}$ be the smallest integer such that $F_{m}^{\left(n_{m}\right)}(0) \neq 0, m=1, \ldots, M$. Then, if $\forall m=1, \ldots, M, n_{m}=n$, then we have:

i. the ratio $\frac{(M n) !}{M^{n M}(n !)^{M}}$ is strictly decreasing in $n$, for any $M>1$, and thus the higher $n$ is, the more important is the advantage of CSI-TR over CSI-R, in term of outage probability,

ii.

$$
\lim _{n \rightarrow \infty} \frac{(M n) !}{M^{n M}(n !)^{M}}= \begin{cases}0, & \text { if } M>1 \\ 1, & \text { if } M=1 .\end{cases}
$$

Proof: First, we note that if $M=1$, the ratio in Corollary 2 is equal to 1 , irrespective of $n$. Now, assume that $M>1$ and

\footnotetext{
${ }^{5}$ The derivation of (53) follows from (111) in Appendix B.
} 
let $V(n)=\frac{(M n) !}{M^{n M}(n !)^{M}}$. Since the bounds in (42) hold for $V(n)$, then we can upper bound $\frac{V(n+1)}{V(n)}$ as follows:

$$
\begin{aligned}
\frac{V(n+1)}{V(n)} & \left.\leq \frac{e}{\sqrt{2 \pi}}\left(\frac{n+1}{e}\right)^{M(n+1)} \frac{\sqrt{2 \pi M(n+}}{((n+1) !}\right)^{M} \\
& =\frac{e}{\sqrt{2 \pi}} \frac{1}{e^{M}} \sqrt{\frac{1+n}{n}}\left(\frac{1+n}{n}\right)^{M n} \\
& \leq \frac{e}{\sqrt{2 \pi}} \frac{1}{e^{M}} \sqrt{2} 2^{M} \\
& =\frac{e}{\sqrt{2 \pi}} e^{\left(M+\frac{1}{2}\right) \log (2)-M} \\
& \leq \frac{e}{\sqrt{2 \pi}} e^{\left(2+\frac{1}{2}\right) \log (2)-2} \\
& =\frac{1}{\sqrt{\pi}} \frac{4}{e} \\
& <1,
\end{aligned}
$$

where (58) follows because the function $x \mapsto \sqrt{\frac{1+x}{x}}\left(\frac{1+x}{x}\right)^{M x}$ is the product of two positive-definite monotonically decreasing functions and thus is also monotonically decreasing; (60) is true because the function $x \mapsto\left(x+\frac{1}{2}\right) \log (2)-x$ is monotonically decreasing and also because $M \geq 2$. Hence $V(n)$ is strictly decreasing in $n$. To prove ii, we upper bound $V(n)$ departing from (49) as follows:

$$
\begin{aligned}
V(n) & \leq \frac{e}{\sqrt{2 \pi}}\left(\frac{n}{e}\right)^{M n} \frac{\sqrt{2 \pi M n}}{(n !)^{M}} \\
& \leq \frac{e}{\sqrt{2 \pi}}\left(\frac{n}{e}\right)^{M n} \frac{\sqrt{2 \pi M n}}{\left(\sqrt{2 \pi n}\left(\frac{n}{e}\right)^{n}\right)^{M}} \\
& =\frac{e}{\sqrt{2 \pi}} \frac{\sqrt{M}}{(2 \pi)^{\frac{M-1}{2}}} \frac{1}{n^{\frac{1}{2}(M-1)}},
\end{aligned}
$$

where (64) is due to the LHS of (41). Since the RHS of (65) converges to 0 as $n \rightarrow \infty$ for any $M>1$, then so does $V(n)$. This completes the proof of Corollary 2.

\section{Performance Limits in the Low-Power Regime Under A Long-Term Power Constraint}

As we highlighted at the end of Section II, the minimum outage probability under LTPC may be derived in a similar way as its counterpart under STPC, but using $s^{*}$ instead of $P$. However, in order for our results in the low power regime to apply under LTPC, one need to verify that when it exists and is finite, $s^{*} \rightarrow 0$ as $P \rightarrow 0$, i.e., $\lim _{P \rightarrow 0} s^{*}(P)=0$. This is true as claimed by Lemma 2 .

Lemma 2: Define the function $\Theta(\cdot, \cdot)$ on $[0, \infty) \times[0, \infty)$ by: $\Theta(P, R)=\underset{\gamma}{\mathrm{E}}\left[\frac{1}{M} \sum_{m=1}^{M} p_{m}^{l t}(\gamma)\right]-P$. For the minimum outage probability of block fading channels described by (1) under LTPC (3), the equivalent STPC $s^{*}(P, R(P))$ defined by (10) is either infinite or converges to 0 as $P \rightarrow 0$. More precisely,:

- If $\exists a>0$ such that $\Theta(P, R(P)) \leq 0, \forall P \in[0, a)$, then $\lim _{P \rightarrow 0} s^{*}(P, R(P))=+\infty$,

- else, $\lim _{P \rightarrow 0} s^{*}(P, R(P))=0$.
Proof: For convenience, the proof is presented in Appendix C.

We note that $\underset{\gamma}{\mathrm{E}}\left[\frac{1}{M} \sum_{m=1}^{M} p_{m}^{l t}(\gamma)\right]$ in Lemma 2 depends on $R$, which itself may scale with the power budget $P$. The case where $R$ does not scale with $P$ may be regarded as a particular case where $R(P)=R$, and thus the first case in Lemma 2 cannot happen. In that case, it holds that $\lim _{P \rightarrow 0} s^{*}(P, R)=0$ by Lemma 2 . On the other hand, if $R$ scales with $P$ such that $\Theta(P, R(P)) \leq 0$ for any $P \in[0, a)$, for some $a>0$, then the equivalent STPC $s^{*}(P, R(P))$ induced by the LTPC $P$ tends toward infinity. As we pointed out in Section II, the latter situation depicts a zero-outage performance itself characterized by delay-limited capacity. We are now ready to state the following result.

Theorem 3: For the block-fading channel described by (1), with perfect CSI at both the transmitter and the receiver, and subject to LTPC (3), the outage performance is characterized as follows:

- If $\exists a>0$ such that $\Theta(P, R(P)) \leq 0, \forall P \in[0, a)$, then $\lim _{P \rightarrow 0} P_{\text {out }}^{l t, C S I-T R}(R(P), P, M)=0$,

- else, $P_{\text {out }}^{l t, C S I-T R}(R(P), P, M) \stackrel{P \rightarrow 0}{\approx} \prod_{m=1}^{M} F_{m}\left(y_{1}\right)$,

where $y_{1}=\frac{e^{2 M R}-1}{M s^{*}}$, where $s^{*}$ is the solution of (10).

Proof: First, assume there exists no $a>0$ such that $\Theta(P, R(P)) \leq 0, \forall P \in[0, a)$, then by Lemma 2 , we have that $s^{*}$ converges to 0 as $P \rightarrow 0 .{ }^{6}$ In this case, along similar lines as in the case of STPC, the lower bound (17) holds true, the only difference is that $P$ is now replaced by $s^{*}$. Thus, we have:

$$
P_{\text {out }}(R, P, M) \geq \prod_{m=1}^{M} F_{m}\left(\frac{2 R}{s^{*}}\right) .
$$

Next, we provide a power policy that satisfies the LTPC and achieves asymptotically the lower bound in (66). For this purpose, let us consider the following power profile:

$$
P_{m}^{*, l t}(\gamma)= \begin{cases}\frac{e^{2 M R}-1}{\gamma_{m}} & \text { if } \gamma_{m}=\max _{1 \leq k \leq M} \gamma_{k} \text { and } \gamma_{m}>\frac{e^{2 M R}-1}{M s^{*}} \\ 0 & \text { otherwise. }\end{cases}
$$

Clearly the power policy given by (67) fulfills the LTPC since:

$$
\begin{aligned}
\underset{\gamma}{\mathrm{E}}\left[\frac{1}{M} \sum_{m=1}^{M} P_{m}^{*, l t}\right] & =\underset{\gamma_{\max }>\frac{e^{2 M R_{-1}}}{M s^{*}}}{\mathrm{E}}\left[\frac{1}{M}\left[\lambda^{l t}(1, \gamma)-\frac{1}{\gamma_{\max }}\right]\right] \\
& \leq \underset{\mathcal{R}\left(s^{*}\right)}{\mathrm{E}}\left[\frac{1}{M} \sum_{m=1}^{M}\left[\lambda^{l t}(b, \gamma)-\frac{1}{\gamma_{m}}\right]\right]^{+} \\
& =P
\end{aligned}
$$

where (69) holds true since (68) only accounts for the power spent over 1 (the strongest) block. Furthermore, this power policy achieves a probability of outage equal to $\operatorname{Prob}\left\{\gamma_{\max } \leq \frac{e^{2 M R}-1}{M s^{*}}\right\}$ which is asymptotically equal to the lower bound in (66).

Second, assume that $\Theta(P, R(P)) \leq 0$ holds on an interval of the form $[0, a)$, with $a>0$, then, again, by Lemma 2, we

\footnotetext{
${ }^{6}$ Although the dependence of $s^{*}$ on $P$ and $R(P)$ should be explicit as it is done in Theorem 3, we make this dependence implicit hereafter for brevity, using $s^{*}$ instead of $s^{*}(P, R(P))$. Analogously, $R$ is used instead of $R(P)$.
} 
have that $s^{*}(P, R(P))$ tends toward $+\infty$ as $P \rightarrow 0$. It follows that the no-outage region $\mathcal{R}\left(s^{*}\right)$ defined by (11) is equal to $\mathbb{R}_{+}^{M}$ which yields an outage probability asymptotically equal to 0 . This completes the proof of Theorem 2 .

We note that in the case where $\lim _{P \rightarrow 0} s^{*}=0$, we still have that $s^{*} \geq P$, i.e., $s^{*}$ converges to 0 slower than $P$. Intuitively, for equal power constraint, an LTPC provides more flexibility than an STPC and hence better performances. Since for bounded $s^{*}$, outage performance under LTPC is similar to that under STPC, but using $s^{*}$ instead of $P$, as asserted by Theorem 3, then one expects $s^{*}$ to be larger than $P$. This can be confirmed as follows. Recalling (9), we have:

$$
\begin{aligned}
P & =\int_{\mathcal{R}\left(s^{*}\right)}\left(\frac{1}{M} \sum_{m=1}^{M} \mu_{m}^{l t}(\gamma)\right) d F(\gamma) \\
& \leq \int_{\mathcal{R}\left(s^{*}\right)} s^{*} d F(\gamma) \\
& =s^{*} \int_{\mathcal{R}\left(s^{*}\right)} d F(\gamma) \\
& \leq s^{*}
\end{aligned}
$$

where (72) follows from the definition of $\mathcal{R}(s)$ in (11), and (74) holds true since the integral in (73) is a probability.

Remark 4: In the case where $s^{*} \rightarrow 0$ as $P \rightarrow 0$, all the results established in Sections III and IV under STPC apply to the LTPC setting, but substituting $P$ by $s^{*}$.

In regard of Lemma 2 and (74), not only $s^{*}$ is larger than $P$, but it may converge asymptotically to infinity as $P \rightarrow 0$. In this case, one can achieve zero-outage performance over a finite number of blocks $M$ while communication at a rate arbitrary close to the delay-limited capacity. The next theorem is an asymptotic characterization of the delay-limited capacity.

Theorem 4: For the block-fading channel described by (1), with perfect CSI at both the transmitter and the receiver, and subject to LTPC (3), the delay-limited capacity $C_{0}(P, M)$ is given by:

$$
C_{0}(P, M) \approx \frac{P}{2 \underset{\gamma}{\mathrm{E}}\left[\gamma_{\text {max }}^{-1}\right]},
$$

provided that $\underset{\gamma}{\mathrm{E}}\left[\gamma_{\max }^{-1}\right]$ is finite. Otherwise, the delay limited capacity is equal to zero.

Proof: Assume that $\underset{\gamma}{\mathrm{E}}\left[\gamma_{\max }^{-1}\right]$ is finite. We split the proof into two parts. A converse part where we show that any zerooutage achievable rate is asymptotically smaller than the RHS of (75). Then, we prove the achievability part.

a) Converse: For a given (asymptotically small) $P$ and a given outage performance $\varepsilon$, let $R$ be the rate, such that the minimum outage probability under LTPC $P$, is equal to $\varepsilon$. We know from Lemma 2 that $s^{*}$, the correspondent equivalent STPC as defined by (10), either converges to infinity or to 0 , as $P \rightarrow 0$. Clearly, the latter case induces a no outage region asymptotically equal to the origin which translates into $R=0$ and thus only the former case is of interest. It is easy to verify that the former case happens if and only if $\Theta(P, R) \leq 0$. Hence, $\exists a>0: \forall P \in[0, a)$, we have:

$$
P \geq \underset{\gamma}{\mathrm{E}}\left[\frac{1}{M} \sum_{m=1}^{M} p_{m}^{l t}(\gamma)\right]
$$

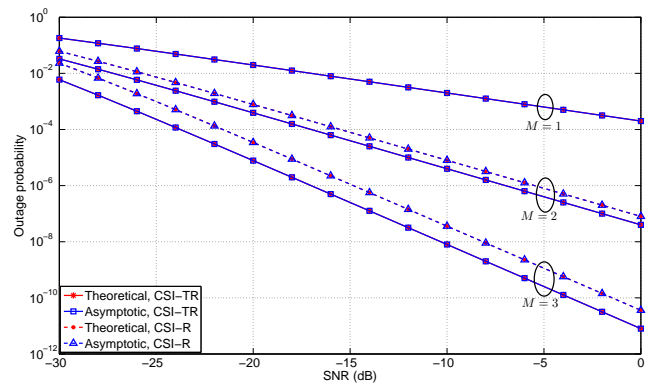

Figure 1. Outage probability versus SNR for Rayleigh fading channels, for different $M$ values, and $R=10^{-4} \mathrm{npcu}$, in the low-power regime.

$$
\geq \underset{\gamma}{\mathrm{E}}\left[\frac{1}{M} \frac{e^{2 M R}-1}{\gamma_{\max }}\right],
$$

which itself implies that:

$$
\begin{aligned}
R & \leq \frac{1}{2 M} \log \left(1+\frac{M P}{\left.\underset{\gamma}{\mathrm{E}\left[\gamma_{\text {max }}^{-1}\right]}\right)}\right. \\
& \approx \frac{P}{2 \underset{\gamma}{\mathrm{E}\left[\gamma_{\text {max }}^{-1}\right]}},
\end{aligned}
$$

from which we establish the converse by taking the limits of (79) as $\epsilon \rightarrow 0$.

b) Achievability: To prove achievability, it suffices to consider the power profile in (67), but with $s^{*}=\infty$ and $R=\frac{P}{2 \underset{\gamma}{2}\left[\gamma_{\max }^{-1}\right]}$. It is then immediate (following the same steps leading to (70)) to see that this power profile is an eligible candidate as it fulfills the LTPC, and that the corresponding outage performance is equal to zero.

Now, assume that $\underset{\gamma}{\mathrm{E}}\left[\gamma_{\max }^{-1}\right]$ is infinite, then by (78), any zerooutage achievable rate is upper-bounded by zero. Hence the delay-limited capacity is equal to zero. This concludes the proof of Theorem 4.

Theorem 4 states that the delay-limited capacity scales linearly with $P$ for a given $M<\infty$. Furthermore, it is immediate to see that the delay-limited capacity increases with $M$ in agreement with the intuition that increasing $M$ provides more flexibility in spending the power and thus does not hurt.

\section{Numerical Results}

First, come our results for the STPC. We present results for normalized i.i.d. Rayleigh and Nakagami-m $(m=2)$ fading channels in Fig. 1 and 2, respectively. Figure 1 shows the outage probability versus $P$ for a given $R$ and for $M=1, M=2$ and $M=3$. For each $M$, Fig. 1 shows the curves corresponding to the exact outage under CSI-TR, its asymptotic expression in Theorem 1, the exact outage under CSI-R and its asymptotic expression given by (28) and (30), respectively. The exact outage under CSI-TR has been obtained by numerical integration of the channel PDF over the outage region given by (7). For $M=1$, the outage probability under CSI-TR and CSI-R always coincide; thus we only display the curves corresponding to CSI-TR in this case. Recall that the SNRs and the rates in 


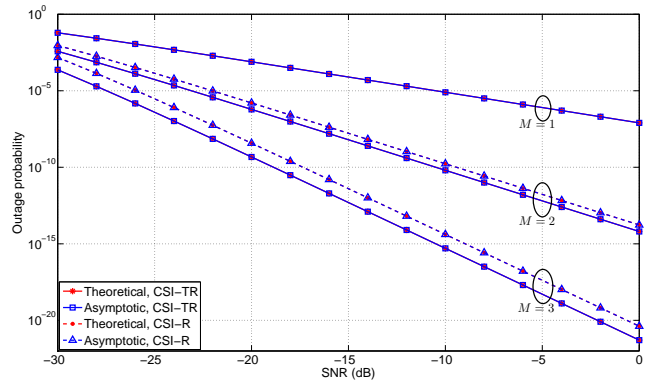

Figure 2. Outage probability versus SNR for Nakagami-m fading channels $(m=2)$, for different $M$ values, and $R=10^{-4} \mathrm{npcu}$, in the low-power regime.

the curves are very low as they correspond to normalized performance measures per degrees of freedom. For instance, in ultra-wideband applications, the bandwidth available is of order of five hundreds mega Hertz $(500 \mathrm{MHz})$. This translates a normalized rate per degrees of freedom $R=10^{-4}$ bits per second per Hertz $(\mathrm{b} / \mathrm{s} / \mathrm{Hz})$ into an aggregate rate of $50 \mathrm{~Kb} / \mathrm{s}$ which is decently high for low-rate applications such as sensor data collection, precision locating and tracking applications. Figure 1 shows the accuracy of our asymptotic analysis since the exact and the asymptotic representations match perfectly. As $M$ increases, the ratio between the outage probabilities under CSI-TR and CSI-R decreases as predicted by Corollary 1. Also, for a given outage performance, the power gain due to CSI-T increases from $0 \mathrm{~dB}$ for $M=1$ to about $1.5 \mathrm{~dB}$ for $M=2$ and reaches $2.18 \mathrm{~dB}$ for $M=3$, exactly as predicted by (54). Finally, we note that the slopes of the curves in Fig. 1 are equal to 1,2 and 3 for $M=1, M=2$ and $M=3$, respectively, since for Rayleigh fading, $n_{m}=1$, as discussed in Remark 2. For Nakagami-m fading, in addition to the above observations, we note first that the slopes of the curves in Fig. 2 are equal to 2, 4 and 6 for $M=1, M=2$ and $M=3$, respectively, since for Nakagami-m $(m=2)$ fading, $n_{m}=2$. Comparing the impact of the two fading on the outage, we observe that for $M=3$ and $P=-4 \mathrm{~dB}$, for instance, the ratio between the outage probabilities with CSI-TR and with CSI-R is equal to about 0.22 in Fig. 1 whereas it is equal to 0.12 in Fig. 2, implying that the advantage of CSI-TR increases with $n_{m}$, again in agreement with Corollary 2 . We note that the asymptotic characterization in Theorem 1 holds true as long as $R \rightarrow 0$, irrespective of the SNR values. The fact that the rate $R$ is set very small in both figures 1 and 2 justifies why the asymptotic curves and the ones obtained by simulations match perfectly well. Should $R$ be chosen not very small, for example $R=0.1 \mathrm{npcu}$, we expect to see a discrepancy between the asymptotic curves and the ones obtained by simulations. This fact is confirmed by Fig. 3. Second, come our results for the LTPC. Figure 4 depicts the outage performance for Rayleigh fading under STPC and LTPC, with $M=2$. In Fig. 4, the rate is set to $R=0.01 \mathrm{npcu}$. Beyond the accuracy of our asymptotic analysis (in Theorems 1 and 3) which curves match perfectly with the exact ones, Fig. 4 highlights the tremendous gain provided by LTPC over STPC even at low-SNR. For instance, at an outage performance equal to $10^{-3}$, the power gain is

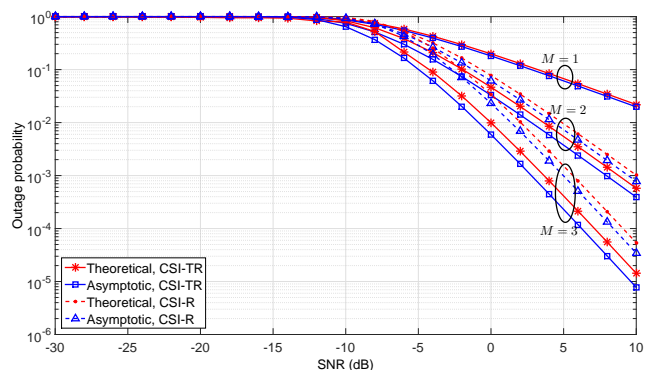

Figure 3. Outage probability versus SNR for Rayleigh fading channels, for different $M$ values, and $R=0.1 \mathrm{npcu}$.

about $14 \mathrm{~dB}$. Of particular interest, is the power corresponding to the delay-limited capacity plotted with a vertical line at $S N R=-15.48 \mathrm{~dB}$. Indeed, this is the power needed to communicate $R=0.01$ nats per channel use (npcu) with zerooutage, again in full agreement with Theorem 4 . In order to verify the increase of the delay-limited capacity with $M$, we depict in Fig. 5 the delay limited capacity versus $S N R$ curves for $M=2$ and $M=3$. Recall that for $M=1$, the delay limited capacity is equal to 0 for Rayleigh fading channels. Likewise, with CSI-R only, the delay limited capacity is also equal to zero. In Fig. 5, the asymptotic curves have been obtained using Theorem 4 whereas the exact curve for $M=2$ has been obtained by solving numerically the following equation [25]:

$P=\left(e^{4 C_{0}}-1\right) \log \left(1+e^{-4 C_{0}}\right)+e^{2 C_{0}}\left(\pi-4 \arctan \left(e^{-2 C_{0}}\right)\right)-4 C_{0}$.

The curve corresponding to exact delay-limited capacity for $M=3$ has been obtained by solving numerically $\Theta\left(P, C_{0}\right)=0$, where $\Theta(\cdot, \cdot)$ is defined in Lemma 2. As can be seen in Fig. 5 , the exact curves and the asymptotic ones match perfectly at low SNR. As SNR increases (above $-10 \mathrm{~dB}$ for $M=2$ and above $-15 \mathrm{~dB}$ for $M=3$ ), the exact and the asymptotic curves are more distinguishable which suggests that the asymptotic analysis does not predict perfectly well the delay-limited capacity behavior in these SNR ranges. However, both the exact curves and the asymptotic ones confirm the capacity gain due to an increase of the frame length $M$. Indeed, increasing $M$ from 2 to 3 provides $60 \%$ capacity increase in the lowpower regime. More interestingly, the delay-limited capacity for $M=3$ is higher than the AWGN capacity at low SNR. This highlights, once again, the benefit of fading in the lowpower regime, even under stringent delay constraints. Finally, we note that as $M \rightarrow \infty$, we expect the delay-limited capacity to converge toward the ergodic capacity with perfect CSI-TR, which is also plotted in Fig. 5. [!t]

\section{Conclusion}

We have studied performance limits of delay-constrained communications with perfect CSI-TR in the low-power regime. Under STPC, we have shown that selection diversity achieves asymptotically the minimum outage performance and provided a simple expression of the outage probability. We have highlighted the gain of CSI-TR over CSI-R only, in terms of outage probability, and shown that this increase 


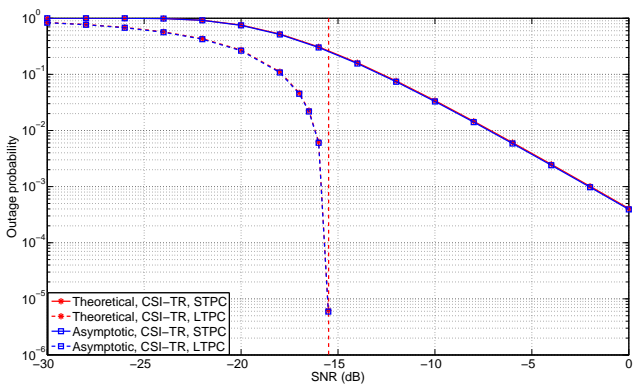

Figure 4. Outage probability versus SNR for Rayleigh fading channels, with $M=2$ and $R=10^{-2} \mathrm{npcu}$, for both STPC and LTPC, in the low-power regime.

grows unboundedly with the delay constraint. Finally, we have discussed the value of CSI-TR for different fading channels in the low-power regime. Subject to LTPC, in addition to the above benefits of fading and CSI-TR, we have characterized the delay-limited capacity and shown that it scales linearly with the power constraint. Selection diversity along with channel inversion are shown to be capacity-achieving in this case.

The present study confirms once again the benefits of communication in the low-power regime. Either in a point-topoint or a multiuser setting, in delay-limited or delay-unlimited communications, the fading along with CSI-TR turn out to be extremely beneficial and provide a substantial gain. Nonetheless, these benefits may be compromised by few technical issues among which the challenging channel estimation and signal decoding in the regime of interest. Circumventing these challenges is certainly not only within our area of expertise, but also depends on advances in microelectronic and signal processing techniques.

\section{APPENDix A}

Proof of the Asymptotic Equality (22)

Assume that $F_{\max }(\cdot)$ admits a non null $n$th derivative at 0 , for some $n>0$. Let $n_{0}$ be the smallest integer such that $F_{\max }^{\left(n_{0}\right)}(0) \neq 0$. Performing Taylor series expansion of $F_{\max }\left(\frac{2 R}{P}\right)$ at the vicinity of $R=0$ to the $n_{0}$ th order yields:

$$
F_{\max }\left(\frac{2 R}{P}\right)=\left(\frac{2}{P}\right)^{n_{0}} F_{\max }^{\left(n_{0}\right)}(0) \frac{R^{n_{0}}}{n_{0} !}+o_{1}\left(R^{n_{0}}\right),
$$

where $o_{1}\left(R^{n_{0}}\right)$ is a function of $R$ such that $\lim _{R \rightarrow 0} \frac{o_{1}\left(R^{n_{0}}\right)}{R^{n_{0}}}=0$. Our objective now is to verify that $F_{\max }\left(\frac{e^{2 M R}-1}{M P}\right)$ can also be written as

$$
F_{\max }\left(\frac{e^{2 M R}-1}{M P}\right)=\left(\frac{2}{P}\right)^{n_{0}} F_{\max }^{\left(n_{0}\right)}(0) \frac{R^{n_{0}}}{n_{0} !}+o_{2}\left(R^{n_{0}}\right),
$$

where $o_{2}\left(R^{n_{0}}\right)$ is a function of $R$ such that $\lim _{R \rightarrow 0} \frac{o_{2}\left(R^{n_{0}}\right)}{R^{n_{0}}}=0$. For this purpose, we note that

$$
\begin{aligned}
& \left.\left(F_{\max }\left(\frac{e^{2 M R}-1}{M P}\right)\right)\right)^{\left(n_{0}\right)} \\
= & \left(\frac{2}{P} e^{2 M R} F_{\max }^{(1)}\left(\frac{e^{2 M R}-1}{M P}\right)\right)^{\left(n_{0}-1\right)}
\end{aligned}
$$

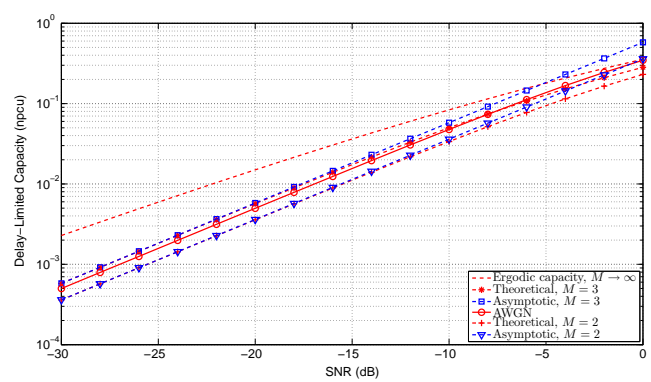

Figure 5. Delay-limited capacity versus SNR for Rayleigh fading channels, with $M=2$ and $M=3$, for LTPC in the low-power regime. For a comparison purpose, the ergodic capacity $(M \rightarrow \infty)$ with full CSI-TR and the AWGN capacity are also plotted.

$$
=\frac{2}{P} \sum_{k=0}^{n_{0}-1}\left(\begin{array}{c}
n_{0}-1 \\
k
\end{array}\right)\left(F_{\max }^{(1)}\left(\frac{e^{2 M R}-1}{M P}\right)\right)^{(k)}\left(e^{2 M R}\right)^{\left(n_{0}-1-k\right)} .
$$

Since all the $k$ th derivative of $F_{\max }(\cdot)$ are null at zero for $k<n_{0}$, only the term $\frac{2}{P}\left(F_{\max }^{(1)}\left(\frac{e^{2 M R}-1}{M P}\right)\right)^{\left(n_{0}-1\right)}$ is of interest. This term, itself can be written as

$$
\begin{aligned}
& \frac{2}{P}\left(F_{\max }^{(1)}\left(\frac{e^{2 M R}-1}{M P}\right)\right)^{\left(n_{0}-1\right)} \\
= & \frac{2}{P}\left(\frac{2}{P} e^{2 M R} F_{\max }^{(2)}\left(\frac{e^{2 M R}-1}{M P}\right)\right)^{\left(n_{0}-2\right)} \\
= & \left(\frac{2}{P}\right)^{2} \sum_{k=0}^{n_{0}-2}\left(\begin{array}{c}
n_{0}-2 \\
k
\end{array}\right)\left(F_{\max }^{(2)}\left(\frac{e^{2 M R}-1}{M P}\right)\right)^{(k)}\left(e^{2 M R}\right)^{\left(n_{0}-2-k\right)} .
\end{aligned}
$$

Again, only the term $\left(\frac{2}{P}\right)^{2}\left(F_{\max }^{(2)}\left(\frac{e^{2 M R}-1}{M P}\right)\right)^{\left(n_{0}-2\right)}$ is of interest since all other terms are equal to zero at $R=0$ as they involve derivatives of $F_{\max }(\cdot)$ to an order smaller than $n_{0}$. It becomes then clear (by induction) that only the term $\left(\frac{2}{P}\right)^{n_{0}} F_{\max }^{\left(n_{0}\right)}\left(\frac{e^{2 M R}-1}{M P}\right)$ evaluated at $R=0$ is not null in the computation of $\left(F_{\max }\left(\frac{e^{2 M R}-1}{M P}\right)\right)^{\left(n_{0}\right)}$ in (84) at $R=0$. That is

$$
\begin{aligned}
\left.\left(F_{\max }\left(\frac{e^{2 M R}-1}{M P}\right)\right)^{\left(n_{0}\right)}\right|_{R=0} & =\left.\left(\frac{2}{P}\right)^{n_{0}} F_{\max }^{\left(n_{0}\right)}\left(\frac{e^{2 M R}-1}{M P}\right)\right|_{R=0} \\
& =\left(\frac{2}{P}\right)^{n_{0}} F_{\max }^{\left(n_{0}\right)}(0) .
\end{aligned}
$$

Applying Taylor series expansion of $F_{\max }\left(\frac{e^{2 M R}-1}{M P}\right)$ at the vicinity of $R=0$ to the $n_{0}$ th order and using (90), we establish (82). To finish the proof, we compute the limit of the ratio between both sides of (22) as follows:

$$
\begin{aligned}
& \lim _{R \rightarrow 0} \frac{F_{\max }\left(\frac{2 R}{P}\right)}{F_{\text {max }}\left(\frac{e^{2 M R}-1}{M P}\right)} \\
= & \lim _{R \rightarrow 0}\left(\frac{F_{\max }\left(\frac{2 R}{P}\right)}{\left(\frac{2}{P}\right)^{n_{0}} F_{\text {max }}^{\left(n_{0}\right)}(0) \frac{R^{n_{0}}}{n_{0} !}} \frac{\left(\frac{2}{P}\right)^{n_{0}} F_{\max }^{\left(n_{0}\right)}(0) \frac{R^{n_{0}}}{n_{0} !}}{F_{\max }\left(\frac{e^{2 M R}-1}{M P}\right)}\right) \\
= & 1,
\end{aligned}
$$

which is the desired result. 


\section{APPENDIX B}

Proof of Theorem 2

We designate by $\mathcal{L}\{f\}$ the Laplace transform of the function $f$. Let $\phi_{m}=\mathcal{L}\left\{f_{m}\right\}, m=1, \ldots, M$. Since $n_{m}$ be the smallest integer such that $F_{m}^{\left(n_{m}\right)}(0) \neq 0$, then there exist $\eta_{m}>0$, such that for any $x \in\left(0, \eta_{m}\right)$, we have:

$$
F_{m}(x)=\frac{F_{m}^{\left(n_{m}\right)}(0)}{n_{m} !} x^{n_{m}}+\mathrm{o}\left(x^{n_{m}}\right),
$$

$m=1, \ldots, M$. Thus, for any $x \in\left(0, \min _{m=1}^{M} \eta_{m}\right)$, we have:

$$
\prod_{m=1}^{M} F_{m}(x) \approx \prod_{m=1}^{M} \frac{F_{m}^{\left(n_{m}\right)}(0)}{n_{m} !} x^{n_{m}} .
$$

Hence, $P_{\text {out }}^{\text {st CSI-TR }}(R, P, M)$ is asymptotically equal to:

$$
\begin{aligned}
P_{\text {out }}^{\text {st } C S I-T R}(R, P, M) & \approx \prod_{m=1}^{M} F_{m}\left(\frac{2 R}{P}\right) \\
& \approx \prod_{m=1}^{M} \frac{1}{M^{n_{m}}} \frac{F_{m}^{\left(n_{m}\right)}(0)}{n_{m} !}\left(\frac{2 M R}{P}\right)^{n_{m}},
\end{aligned}
$$

where (97) is a consequence of (95) and the fact that as $P_{\text {out }}^{s t, C S I-T R}(R, P, M) \rightarrow 0$, then we necessarily have $\frac{R}{P} \rightarrow 0$. Before computing $P_{\text {out }}^{s t, C S I-R}(R, P, M)$, we note first that since $F_{m}^{\left(n_{m}\right)}(0) \neq 0$, then we have:

$$
\begin{aligned}
0 & \neq F_{m}^{\left(n_{m}\right)}(0) \\
& =\lim _{x \rightarrow 0} F_{m}^{\left(n_{m}\right)}(x) \\
& =\lim _{s \rightarrow \infty} s \mathcal{L}\left\{F_{m}^{\left(n_{m}\right)}\right\}(s) \\
& =\lim _{s \rightarrow \infty} s\left(s^{n_{m}} \mathcal{L}\left\{F_{m}\right\}(s)-\sum_{k=1}^{n_{m}} s^{k-1} F_{m}^{\left(n_{m}-k\right)}(0)\right) \\
& =\lim _{s \rightarrow \infty} s s^{n_{m}} \frac{1}{s} \mathcal{L}\left\{f_{m}\right\}(s) \\
& =\lim _{s \rightarrow \infty} s^{n_{m}} \phi_{m}(s\}
\end{aligned}
$$

where (100) follows from the Initial Value Theorem; (101) holds true by applying the Laplace transform rule of the $n_{m}$-th derivative and (102) is due to the fact that $n_{m}$ is the smallest integer such that $F_{m}^{\left(n_{m}\right)}(0) \neq 0$ and also by applying the Laplace transform rule of the integral. That is $\lim _{s \rightarrow \infty} s^{n_{m}} \phi_{m}(s\}$ exists and is non-null. We are now ready to compute $P_{\text {out }}^{\text {st,CSI-R }}(R, P, M)$. Let $n$ be the smallest integer such that $F_{\Sigma}^{(n)}(0) \neq 0$, then again there exists $\eta>0$, such that for any $x \in(0, \eta)$, we have:

$$
F_{\Sigma}(x)=\frac{F_{\Sigma}^{(n)}(0)}{n !} x^{n}+o\left(x^{n}\right) .
$$

We compute $F_{\Sigma}^{(n)}(0)$ as follows:

$$
\begin{aligned}
F_{\Sigma}^{(n)}(0) & =\lim _{x \rightarrow 0} F_{\Sigma}^{(n)}(x) \\
& =\lim _{s \rightarrow \infty} s^{n} \mathcal{L}\left\{f_{\Sigma}\right\}(s) \\
& =\lim _{s \rightarrow \infty} s^{n} \prod_{m=1}^{M} \phi_{m}(s) \\
& =\lim _{s \rightarrow \infty} \frac{s^{n}}{s^{\sum_{m=1}^{M} n_{m}}} \prod_{m=1}^{M} s^{n_{m}} \phi_{m}(s),
\end{aligned}
$$

where (106) is justified exactly as (102), (107) follows because the PDF of $\sum_{m=1}^{M} \gamma_{m}$ is given by $f_{\Sigma}(x)=\left(f_{1} * \ldots * f_{M}\right)(x)$, where $*$ designates the convolution operator; and (108) holds true since both limits in (103) and (107) exist. Thus, the limit in (108) exists and is non-null (since $F_{\Sigma}^{(n)}(0) \neq 0$ ). But, $\lim _{s \rightarrow \infty} \prod_{m=1}^{M} s^{n_{m}} \phi_{m}(s) \neq 0$ due to (103). Hence

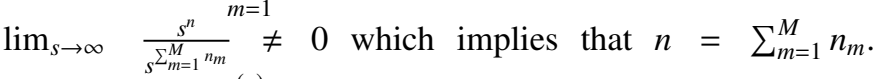
Consequently, $F_{\Sigma}^{(n)}(0)$ can be further simplified as:

$$
\begin{aligned}
F_{\Sigma}^{(n)}(0) & =\lim _{s \rightarrow \infty} \prod_{m=1}^{M} s^{n_{m}} \phi_{m}(s) \\
& =\prod_{m=1}^{M} F_{m}^{\left(n_{m}\right)}(0),
\end{aligned}
$$

where (110) is obtained from (109) using both (103) and (98). Combining (30), (104) and (110), the outage probability with CSI at the receiver can be expressed by:

$$
P_{\text {out }}^{s t, C S I-R}(R, P, M) \approx \frac{\prod_{m=1}^{M} F_{m}^{\left(n_{m}\right)}(0)}{\left(\sum_{m=1}^{M} n_{m}\right) !}\left(\frac{2 M R}{P}\right)^{\sum_{m=1}^{M} n_{m}} .
$$

Finally, using (97) and (111), we have:

$$
\frac{P_{\text {out }}^{s t, C S I-T R}(R, P, M)}{P_{\text {out }}^{s t, C S I-R}(R, P, M)} \approx \frac{1}{M^{\sum_{m=1}^{M} n_{m}}} \frac{\left(\sum_{m=1}^{M} n_{m}\right) !}{\prod_{m=1}^{M} n_{m} !},
$$

which we wanted to prove.

\section{APPENDIX C}

\section{Proof of Lemma 2}

First, we note that $\mathcal{P}(s)$ defined in (10) is strictly increasing on $[0, \infty)$. Indeed, let $s_{1}$ and $s_{2}$ be two nonnegative real numbers, such that $s_{1}>s_{2}$. Then we have:

$$
\begin{aligned}
\mathcal{P}\left(s_{1}\right)-\mathcal{P}\left(s_{2}\right) & =\int_{\mathcal{R}\left(s_{1}\right) \cap \mathcal{R}^{c}\left(s_{2}\right)} \frac{1}{M} \sum_{m=1}^{M} p_{m}^{l t}(\gamma) d F(\gamma) \\
& >\int_{\mathcal{R}\left(s_{1}\right) \cap \mathcal{R}^{c}\left(s_{2}\right)} s_{2} d F(\gamma) \\
& =s_{2} \operatorname{Prob}\left\{\gamma \in \mathcal{R}\left(s_{1}\right) \cap \mathcal{R}^{c}\left(s_{2}\right)\right\} \\
& \geq 0,
\end{aligned}
$$

as we claimed. In the above derivation, (113) follows because $\mathcal{R}\left(s_{2}\right) \subset \mathcal{R}\left(s_{1}\right)$ since $s_{1}>s_{2} ;(114)$ holds true since $\gamma \in \mathcal{R}^{c}\left(s_{2}\right)$ and thus $s_{2}<\frac{1}{M} \sum_{m=1}^{M} p_{m}^{l t}(\gamma)$ and also because $\mathcal{R}\left(s_{1}\right) \cap \mathcal{R}^{c}\left(s_{2}\right)$ is a set of positive measure (in the sense of Lebesgue).

Second, for $s=0, \mathcal{R}(0)=\emptyset$, the empty set, and thus $\mathcal{P}(0)=0$. Finally, we verify the continuity of $\mathcal{P}(s)$ on $[0, \infty)$ as follows. First, we write $\mathcal{P}(s)$ as follows:

$$
\mathcal{P}(s)=\int_{\mathbb{R}_{+}^{M}}\left(\frac{1}{M} \sum_{m=1}^{M} p_{m}^{l t}(\gamma)\right) \chi_{\mathcal{R}(s)}(\gamma) d F(\gamma),
$$

where $\chi_{\mathcal{R}(s)}(\gamma)$ is the indicator function of $\mathcal{R}(s)$. Then, we notice that the function $g(\cdot, \cdot)$ defined on $\mathbb{R}_{+}^{M} \times(0, \infty)$ by: $g(\gamma, s) \triangleq \frac{1}{s}\left(\frac{1}{M} \sum_{m=1}^{M} p_{m}^{l t}(\gamma)\right) \chi_{\mathcal{R}(s)}(\gamma) f(\gamma)$ is continuous in $s$ for every $\gamma$. Furthermore,

$$
|g(\gamma, s)| \leq \frac{1}{s} s \chi_{\mathcal{R}(s)} f(\gamma)
$$




$$
\begin{aligned}
& =\chi_{\mathcal{R}(s)} f(\gamma) \\
& \leq f(\gamma),
\end{aligned}
$$

and $\int_{\mathbb{R}_{+}^{M}} d F(\gamma)=1 \leq \infty$. Hence, by The Parameter-Dependent Integral Theorem, $\frac{\mathcal{P}(s)}{s}$ is continuous in $s$, for all $s \in(0, \infty)$. It follows that $\mathcal{P}(s)$, being the product of two continuous functions, is also continuous on $(0, \infty)$. It only remains to verify the continuity of $\mathcal{P}(s)$ at 0 . For that, we recall from the above derivation, the following bound:

$$
\begin{aligned}
|\mathcal{P}(s)| & \leq \int_{\mathbb{R}_{+}^{M}} s \chi_{\mathcal{R}(s)}(\gamma) d F(\gamma) \\
& \leq s .
\end{aligned}
$$

By taking the limits on both sides of (122), we obtain that $\lim _{s \rightarrow 0} \mathcal{P}(s)=0$, which is equal to $\mathcal{P}(0)$. That is, $\mathcal{P}(s)$ is also continuous at 0 .

In summary, we have verified that $\mathcal{P}(s)$ is a strictly increasing and continuous function on $[0, \infty)$, with $\mathcal{P}(0)=0$. To complete the proof, it suffices to recall that $s^{*}(P)$ satisfies (10) and distinguish two cases:

Case 1: $P<\lim _{s \rightarrow \infty} \mathcal{P}(s)=\underset{\gamma}{\mathrm{E}}\left[\frac{1}{M} \sum_{m=1}^{M} p_{m}^{l t}(\gamma)\right]$

In this case, $s^{*}$ satisfies $\mathcal{P}\left(s^{*}(P)\right)=P$ due to the continuity of $\mathcal{P}(s)$. Applying the limit when $P \rightarrow 0$ on both sides of the previous equality and using the fact that $\mathcal{P}(s)$ is strictly increasing along with the fact that $\mathcal{P}(0)=0$, we obtain $\lim _{P \rightarrow 0} s^{*}(P)=0$.

$$
\text { Case 2: } P \geq \underset{\gamma}{\mathrm{E}}\left[\frac{1}{M} \sum_{m=1}^{M} p_{m}^{l t}(\gamma)\right]
$$

In this case, it is clear that $s^{*}=\sup \{s: \mathcal{P}(s)<P\}=+\infty$, since $\mathcal{P}(s)$ is continuous and increasing on $[0, \infty)$.

This completes the proof of Lemma 2.

\section{REFERENCES}

[1] S. Verdu, "On channel capacity per unit cost," IEEE Trans. Inform. Theory, vol. 36, no. 5, pp. 1019-1030, 1990.

[2] A. Lozano, A. Tulino, and S. Verdu, "Multiple-antenna capacity in the low-power regime," IEEE Trans. Inform. Theory, vol. 49, no. 10, pp. 2527-2544, Oct. 2003.

[3] C. Luo, M. Medard, and L. Zheng, "On approaching wideband capacity using multitone FSK," IEEE Journal on Selected Areas in Communications, vol. 23, no. 9, pp. 1830-1838, 2005.

[4] M. Gursoy, "MIMO wireless communications under statistical queueing constraints," IEEE Trans. Inform. Theory,, vol. 57, no. 9, pp. 5897 5917, Sep. 2011.

[5] S. Loyka and G. Levin, "Diversity-multiplexing tradeoff in the low-SNR regime," IEEE Communications Letters, vol. 15, no. 5, pp. 542-544, 2011.

[6] L. Yang and G. Giannakis, "Ultra-wideband communications: an idea whose time has come," IEEE Signal Processing Magazine, vol. 21, no. 6, pp. 26-54, 2004.

[7] D. Feng, C. Jiang, G. Lim, L. Cimini, Jr., G. Feng, and G. Li, "A survey of energy-efficient wireless communications," IEEE Communications Surveys $\mathcal{E}$ Tutorials, vol. 15, no. 1, pp. 167-178, First Quarter 2013.

[8] S. Verdu, "Spectral efficiency in the wideband regime," IEEE Trans. Inform. Theory, vol. 48, no. 6, pp. 1319-1343, Jun. 2002.

[9] L. Zheng, N. C. D. Tse, and M. Médard, "Channel coherence in the lowSNR regime," IEEE Trans. Inform. Theory, vol. 53, no. 3, pp. 976-997, Mar. 2007.

[10] S. Ray, M. Medard, and L. Zheng, "On noncoherent MIMO channels in the wideband regime: Capacity and reliability," IEEE Trans. Inform. Theory, vol. 53, no. 6, pp. 1983-2009, Jun. 2007.

[11] S. Borade and L. Zheng, "Wideband fading channels with feedback," IEEE Trans. Inform. Theory, vol. 56, no. 12, pp. 6058-6065, Dec. 2010.
[12] C. Zhong, S. Jin, K.-K. Wong, M.-S. Alouini, and T. Ratnarajah, "Low SNR capacity for MIMO Rician and Rayleigh-Product fading channels with single co-channel interferer and noise," IEEE Trans. Commun., vol. 58, no. 9, pp. $2549-2560$, Sep. 2010.

[13] X. Li, S. Jin, M. R. McKay, X. Gao, and K. K. Wong, "Capacity of MIMO-MAC with transmit channel knowledge in the low SNR regime," IEEE Trans. Wireless Commun., vol. 9, no. 3, pp. 926-931, Mar. 2010.

[14] A. Host-Madsen, M. Uppal, and Z. Xiong, "On outage capacity in the low power regime," IEEE Trans. Inform. Theory, vol. 58, no. 2, pp. 888-896, 2012

[15] R. Negi and J. Cioffi, "Delay-constrained capacity with causal feedback," IEEE Trans. on Inform. Theory, vol. 48, no. 9, pp. 2478-2494, Sep 2002.

[16] S. Dey and J. Evans, "Outage capacity and optimal power allocation for multiple time-scale parallel fading channels," IEEE Trans. on Wireless Communications, vol. 6, no. 7, pp. 2369-2373, July 2007.

[17] E. Jorswieck and H. Boche, "Delay-limited capacity: multiple antennas, moment constraints, and fading statistics," IEEE Trans. on Wireless Communications, vol. 6, no. 12, pp. 4204-4208, December 2007.

[18] A. Lozano, A. Tulino, and S. Verdu, "Optimum power allocation for parallel Gaussian channels with arbitrary input distributions," IEEE Trans. on Inform. Theory, vol. 52, no. 7, pp. 3033-3051, July 2006.

[19] J. Luo, R. Yates, and P. Spasojevic, "Service outage based power and rate allocation for parallel fading channels," IEEE Trans. on Inform. Theory, vol. 51, no. 7, pp. 2594-2611, July 2005.

[20] E. Biglieri, J. Proakis, and S. Shamai, "Fading channels: Informationtheoretic and communications aspects," IEEE Trans. Inform. Theory, vol. 44, no. 6, pp. 2619-2692, Oct. 1998.

[21] A. Avestimehr and D. Tse, "Outage capacity of the fading relay channel in the low-snr regime," IEEE Trans. Inform. Theory, vol. 53, no. 4, pp. $1401-1415,2007$.

[22] D. Tse and P. Viswanath, Fundamentals of wireless communication. New York, NY, USA: Cambridge University Press, 2005.

[23] Z. Rezki and M.-S. Alouini, "On the outage capacity of the block fading channel at low-power regime," in in Proc. IEEE International Sysmposium on Information Theory (ISIT'2014), Hanolulu, HI, USA, Jun. 2014, pp. 2894-2898.

[24] L. H. Ozarow, S. Shamai, and A. D. Wyner, "Information theoretic considerations for cellular mobile radio," IEEE Trans. Veh. Technol., vol. 43, no. 2, pp. 359-378, May 1994.

[25] G. Caire, G. Taricco, and E. Biglieri, "Optimum power control over fading channels," IEEE Trans. Inform. Theory, vol. 45, no. 5, pp. 1468 -1489 , Jul. 1999

[26] F. W. J. Olver, D. W. Lozier, R. F. Boisvert, and C. W. Clark, Eds., NIST Handbook of Mathematical Functions. New York, NY: Cambridge University Press, 2010.

[27] M. Abramowitz and I. Stegun, Handbook of Mathematical Functions with Formulas, Graphs, and Mathematical Tables, ser. Applied mathematics series. U.S. Govt. Print. Off., 1964, no. v. 55, no. 1972.

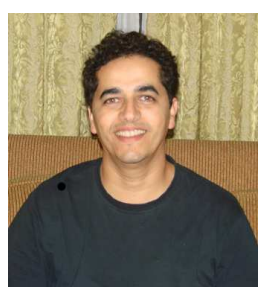

Zouheir Rezki (S'01, M'08, SM'13) was born in Casablanca, Morocco. He received the Diplôme d'Ingénieur degree from the École Nationale de l'Industrie Minérale (ENIM), Rabat, Morocco, in 1994, the M.Eng. degree from École de Technologie Supérieure, Montreal, Québec, Canada, in 2003, and the Ph.D. degree from École Polytechnique, Montreal, Québec, in 2008, all in electrical engineering. From October 2008 to September 2009, he was a postdoctoral research fellow with Data Communications Group, Department of Electrical and Computer Engineering, University of British Columbia. He is now a Senior Research Scientist at King Abdullah University of Science and Technology (KAUST), Thuwal, Mekkah Province, Saudi Arabia. His research interests include: security of data networks, performance limits of communication systems, cognitive and sensor networks, optical communications and low-complexity detection algorithms. 


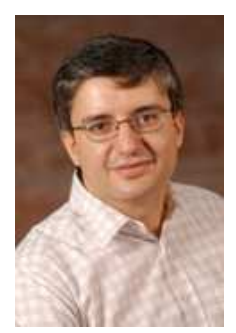

Mohamed-Slim Alouini (S'94, M'98, SM'03, F'09) was born in Tunis, Tunisia. He received the Ph.D. degree in Electrical Engineering from the California Institute of Technology (Caltech), Pasadena, CA, USA, in 1998. He served as a faculty member in the University of Minnesota, Minneapolis, MN, USA, then in the Texas A\&M University at Qatar,
Education City, Doha, Qatar before joining King Abdullah University of Science and Technology

(KAUST), Thuwal, Makkah Province, Saudi Arabia as a Professor of Electrical Engineering in 2009. His current research interests include the modeling, design, and performance analysis of wireless communication systems. 